\title{
674 IL-27 SIGNALING DRIVES A TYPE 1 INTERFERON-LIKE GENE EXPRESSION PROGRAM OF IMMUNOREGULATORY PATHWAYS ASSOCIATED WITH CANCER PROGRESSION
}

Jonathan Hill*, Devapregasan Moodley, Jing Hua, Kerry White, Christine Miller, Secil Koseoglu, Ricard Masia, Benjamin Lee, Vito Palombella. Surface Oncology, Inc., Cambridge, MA, United States

Background Interleukin (IL)-27 is a heterodimeric immunoregulatory cytokine that signals through the JAK/STAT pathway to increase the expression of coinhibitory receptors on immune cells (e.g. PD-L1, TIM-3, LAG-3) and dampen inflammatory cytokine production. Blockade of IL-27 leads to antitumor activity in preclinical mouse models of lung metastases. A Phase 1 trial of SRF388 (NCT04374877), a first-inclass anti-IL-27 antibody, has demonstrated monotherapy antitumor activity in a patient with non-small cell lung cancer (NSCLC). ${ }^{1}$ The current study aimed to characterize the immunoregulatory impact of IL-27 signaling by gene expression profiling.

Methods Gene expression changes induced by IL-27 were examined in activated human $\mathrm{CD} 4+\mathrm{T}$ cells, human peripheral blood mononuclear cells (PBMCs), and the IL-27RA-expressing lung cancer cell line NCI-H2228 by microarray or single cell RNA-sequencing. The resulting IL-27 signature genes were interrogated by Gene Set Enrichment Analysis (GSEA) using publicly available datasets, including single cell RNA-seq analysis of the tumor microenvironment, from patients with NSCLC. ${ }^{2}$

Results IL-27 induced a robust gene expression program in human immune cells that included several inhibitory receptors and canonical interferon regulated genes such as guanylatebinding proteins and interferon regulatory factors. GSEA and interferon signature analysis showed a striking overlap with those genes regulated by interferon-beta, a cytokine known to drive immune suppression associated with chronic viral infection and that is used therapeutically for controlling inflammation associated with the autoimmune disease multiple sclerosis. Moreover, interferon regulated pathways have recently emerged as a mechanism of resistance to immune checkpoint blockade in cancer. Exploration of the IL-27 gene signature in published datasets showed enrichment in macrophage populations associated with progressive disease in patients with NSCLC. While many of the properties of IL-27-mediated immune regulation have focused on hematopoietic cells, IL27RA is also expressed on tumor cells from NSCLC patients with progressive disease as well as lung cancer cell lines in which IL-27 can upregulate PD-L1, IDO1, and other canonical interferon regulated genes.

Conclusions These studies elucidate the transcriptional networks that are engaged after IL-27 signaling in immune and cancer cells and highlight the parallels with interferon-associated immune regulation. Blockade of IL-27 provides a novel therapeutic strategy to alleviate a gene transcriptional program implicated in immune suppression and checkpoint resistance.

\section{REFERENCES}

1. Patnaik A, Morgensztern D, Mantia C, et al. Results of a phase 1 study of SRF388, a first-in-human, first-in-class, high-affinity anti-IL-27 antibody in advanced solid tumors. J Clin Oncol 2021;39:2551-2551.

2. Maynard A, McCoach CE, Rotow JK, et al. Therapy-induced evolution of human lung cancer revealed by single-cell RNA sequencing. Cell 2020;182:1232-1251.

http://dx.doi.org/10.1136/jitc-2021-SITC2021.674 